\title{
Synthetic tests of geoid-viscosity inversion: A layered viscosity case
}

\author{
Motoyuki Kido ${ }^{1}$ and Satoru Honda ${ }^{2}$ \\ ${ }^{1}$ Ocean Research Institute, University of Tokyo, 164-8639, Japan \\ ${ }^{2}$ Department of Earth and Planetary System Science, University of Hiroshima, 739-8526, Japan
}

(Received April 2, 1998; Revised November 2, 1998; Accepted November 5, 1998)

\begin{abstract}
We revisited the resolving power of viscosity inversion in terms of geoid misfit in a 2-D Cartesian geometry under the assumption that the mantle viscosity is laterally stratified. Firstly, we considered a simple case of two viscosity layers only, which is described by two parameters of the amount and the depth of the viscosity jump. The uniqueness of the inversion was examined by evaluating misfits between the reference geoid for a reference viscosity and that for a viscosity described by the changing two parameters. The misfits are mapped into 2-D model space as a function of the two parameters. Three types of density distribution are tested; they are vertically constant (1), taken from a tomographic model (2), and the same but includes artificial noise (3). We found that, at least for this simple case, the viscosity solution keeps unique in the entire 2-D model space using whole degree band (1-8) of geoid. This holds even if the artificial noise is rather large (70\%), though the solution is slightly different from the reference viscosity. However, we also observed non-uniqueness, such as trade-off between the two parameters, when individual degree components of geoid are concerned. In the next, we employed a more realistic viscosity structure, having seven iso-viscous layers. It is no longer possible to describe 6-D model space easily. Therefore we tried to reconstruct a reference viscosity from the reference geoid using genetic algorithm search. According to this analysis, nearly the same solution with the reference viscosity can be reconstructed, while solutions apart from the reference viscosity with increase of noise in the density distribution.
\end{abstract}

\section{Introduction}

Over the last decade, a great number of studies has been made to investigate the mantle viscosity profile inferred from observed geoid and seismic tomographic data (e.g., Richards and Hager, 1984). Currently there is a great progress in developing new methods to analyze the mantle viscosity with more realistic conditions, taking compressibility (Forte and Peltier, 1991; Thoraval et al., 1994; Corrieu et al., 1995; Panasyuk et al., 1996), lateral viscosity variation (Ricard et al., 1988; Richards and Hager, 1989; Ricard et al., 1991; Ribe, 1992; Zhang and Christensen, 1993; Forte and Peltier, 1994), nonlinear rheology (Čadek et al., 1993), and the $660 \mathrm{~km}$ discontinuity (Thoraval et al., 1995; Čadek et al., 1997; Forte and Woodward, 1997; Wen and Anderson, 1997) into account. The accuracy of tomographic models has also been improved significantly. However, most of the studies present quite different viscosity profiles, and there is no agreement except that the lower mantle would be higher viscous than the upper mantle.

This discrepancy mainly comes from the used density model. Usually density models are simply converted from velocity anomalies derived from tomographic models using a linear velocity-density relation, which can be a function of depth. On the contrary, some gropes employ a priori density anomalies for a certain part of the mantle. For two typical studies, see King and Masters (1992) and Hager and Richards (1989) for example. While the former converted

Copy right (C) The Society of Geomagnetism and Earth, Planetary and Space Sciences (SGEPSS); The Seismological Society of Japan; The Volcanological Society of Japan; The Geodetic Society of Japan; The Japanese Society for Planetary Sciences. velocity anomalies into a density model for the entire mantle, the latter imposed high density anomalies in the upper mantle associated with subducting slabs where low velocity anomalies are observed in long wavelength tomographic models. These two density models are much different in the shallow upper mantle, and these force the obtained viscosity profiles are much different as well. King and Masters (1992) prefer low viscous transition zone, while Hager and Richards (1989) suggest a low viscous asthenosphere. Further work has been made to construct more realistic density models, correcting for continental roots (Forte et al., 1995; Doin et al., 1996), using various geological data (Ricard et al., 1995), simulating past subduction history (Ricard et al., 1993). In spite of these studies and improvements of seismic tomography results, large uncertainties in estimating density structure still remain due to the difficulty of interpreting the seismic velocity anomaly beneath cratons (Jordan, 1978) and subduction zones (Karato, 1995).

Another reason for the existence of many different viscosity profiles is the progress in the inversion methods. Traditional inversion techniques, such as calculating partial derivatives (e.g., Tarantola and Valette, 1982), tend to find solutions strongly depending on a starting model. Recent studies using this type of inversion have been improved by introducing a penalty for the smoothness of the solution instead for the distance from the starting model (King and Masters, 1992; Forte et al., 1994; Mitrovica and Forte, 1997). On the contrary, Monte Carlo inversions (Ricard et al., 1989) can search the entire model space at the expense of much computation time. Therefore only several free parameters can be allowed in it. Recently, a much more efficient inversion method, 
called genetic algorithm (GA), has been introduced to the Earth science community (e.g., Sen and Stoffa, 1992). GA has the merit of Monte Carlo search, but drastically saves computation time using dynamical weighting or probability of searching area in the model space. Details of the general GA method can be found in Goldberg (1989). The GA has first been applied to the geoid-viscosity inversion problem by King (1995). He demonstrated the applicability of GA, but also found multiple viscosity solutions even using the same density model. Kido and Čadek (1997) also applied GA to this problem but for regional intermediate wavelength geoid analysis, and found multiple solutions for regions where the resolution of the tomographic model is poor. In both studies multiple solutions are quite different to one another, which indicates the non-uniqueness in this kind of inversion.

In this study, we examine the potential resolving power or uniqueness of the geoid-viscosity inversion in a 2-dimensional Cartesian geometry. Moreover we also estimate the effect of noise in the density distribution on the resolving power. At first, in Section 2, we show the nature of the uniqueness of the geoid-viscosity inversion using a viscosity variation simply depending on two parameters. Uniqueness of the problem is discussed based on maps representing the 2-D model space of misfit between the geoid for a reference viscosity and that for varying viscosities. In Section 3, we employed a more realistic case that viscosity can depend on six parameters. Here we conduct synthetic inversions using GA to reconstruct the reference viscosity instead of mapping the entire model space like in the case of two parameters. In both cases, we also test how noise in the density distribution devaluate the resolving power of the inversion, which corresponds to uncertainties in the interpretation of seismic velocity anomalies.

\section{A Case for Two Parameters}

In this section the complete morphology of 2-D model space will be shown for two layers of viscosity variation. At first, we describe the method of calculating the geoid and density distributions used in this study.

Geoid anomaly represents perturbation of the gravity potential field, which consists of the integral of density anomalies in the mantle (internal loads) and the deflection of all possible density boundaries, e.g. Earth's surface and the coremantle boundary (CMB). These boundary deflections are induced by the mantle flow, which is driven by the internal loads. The instantaneous mantle flow can be calculated when viscosity structure in the mantle is given. The basic equations are the equation of motion, continuity equation, constitutive law, and Poisson's equation. Assuming a simple model, such as no lateral viscosity variation and Newtonian mantle rheology, these equations can be separately solved for each wavelength component analytically. Then the resultant geoid can easily be calculated using the propagator matrix method. This method is described in Hager and Clayton (1989) in detail. In this study, we assume a non self-gravitating incompressible fluid lies in a 2-D Cartesian geometry whose aspect ratio is 8 . Boundary conditions are free slip and no vertical motion at the top and bottom, and periodical at the both sides. These assumptions lead to a rather simple approximation of the Earth's mantle, therefore the predicted geoid may significantly differ from the observational geoid. However this fact does not prohibit to examine the potential resolving power of the geoid-viscosity inversion, which is the goal of this paper. This is because reference geoid is also calculated under the same conditions as geoid to be compared.

We use four density distributions in this study. The first one, here called CON, has no vertical density variation and has only lateral variation assigned that all coefficients in its Fourier expansion will be 1 . This density distribution is far from the Earth, however, is suitable to reveal nature of the potential resolving power of the inversion. The second density distribution, we call it TOM, is taken from an equatorial cross section of a recent tomographic model result for Swave velocity ( $\mathrm{Li}$ and Romanowicz, 1996). We horizontally scaled the original cross section whose aspect ratio is 13.8 to aspect ratio 8 in order to have the same conditions with the density distribution CON. S-wave velocity anomalies are converted to density anomalies assuming $\delta \rho / \delta v=0.2$ $\left(\mathrm{kg} \cdot \mathrm{m}^{-3}\right) /\left(\mathrm{m} \cdot \mathrm{s}^{-1}\right)$, where $\delta \rho$ is density anomaly and $\delta v$ velocity anomaly. This tomography derived density distribution is illustrated in Fig. 1. Stream lines, expected geoid, and boundary deformations for a reference viscosity are also drawn in the figure. It simply has one viscosity jump of two order of magnitude at the depth of $660 \mathrm{~km}$. We also prepare the other density distribution $\rho_{\text {noise }}$, which is derived by adding artificial noise to TOM. TOM is taken from the equatorial cross section and can be denoted as $\rho_{\mathrm{e}}(x, z)$, where $x$ and $z$ are horizontal and vertical coordinates respectively. Here, the noise is also taken from the tomographic model but from a cross section at the meridian and denoted as $\rho_{\mathrm{m}}(x, z)$. Then the density model including noise used in this study $\rho_{\text {noise }}(x, z)$ is defined as

$$
\rho_{\text {noise }}(x, z)=(1-r) \cdot \rho_{\mathrm{e}}(x, z)+r \cdot \rho_{\mathrm{m}}(x, Z-z),
$$

where $r$ is a constant and $Z$ the height of the mantle to be considered. As is defined in Eq. (1), the noise density $\rho_{\mathrm{m}}(x, z)$ has been turned upside down before the addition in order to be as random as possible relative to $\rho_{\mathrm{e}}(x, z)$. We employed two noise levels of density distributions T30 and T70. The $r$ is set to $0.3 /(1+0.3)=0.231$ and $0.7 /(1+0.7)=0.412$ so that ratio of amplitude of the noise to the original density will be $30 \%$ and $70 \%$, respectively. All the density distribution used here are truncated up to degree 8 in the Fourier series.

As shown by the right hand small drawing in Fig. 1, the reference viscosity increases by a factor of 100 at the depth $660 \mathrm{~km}$. The reference geoids corresponding to the reference viscosity calculated under the conditions described above are drawn in the top part of Fig. 1.

Then we calculated geoids, which will be compared to the reference geoid. As mentioned above, the used viscosity profile is a step function, which has two parameters; the depth of jump $z_{\text {jump }}$ and ratio of upper to lower part of mantle viscosity $\eta_{\text {upp }} / \eta_{\text {low }}$. Using these description, the reference viscosity can be represented that $z_{\text {jump }}=$ $660 \mathrm{~km}$ and $\log _{10}\left(\eta_{\text {upp }} / \eta_{\text {low }}\right)=-2$. We calculated geoids for viscosity model space that $z_{\text {jump }}$ from 0 to $2900 \mathrm{~km}$ and $\log _{10}\left(\eta_{\text {upp }} / \eta_{\text {low }}\right)$ from -4 to 0 .

Thus calculated geoid $\mathrm{cal} N$ is compared with the reference geoid ${ }_{\text {ref }} N$ for the reference viscosity. Root mean squares (RMS) of their difference normalized by RMS amplitude of 


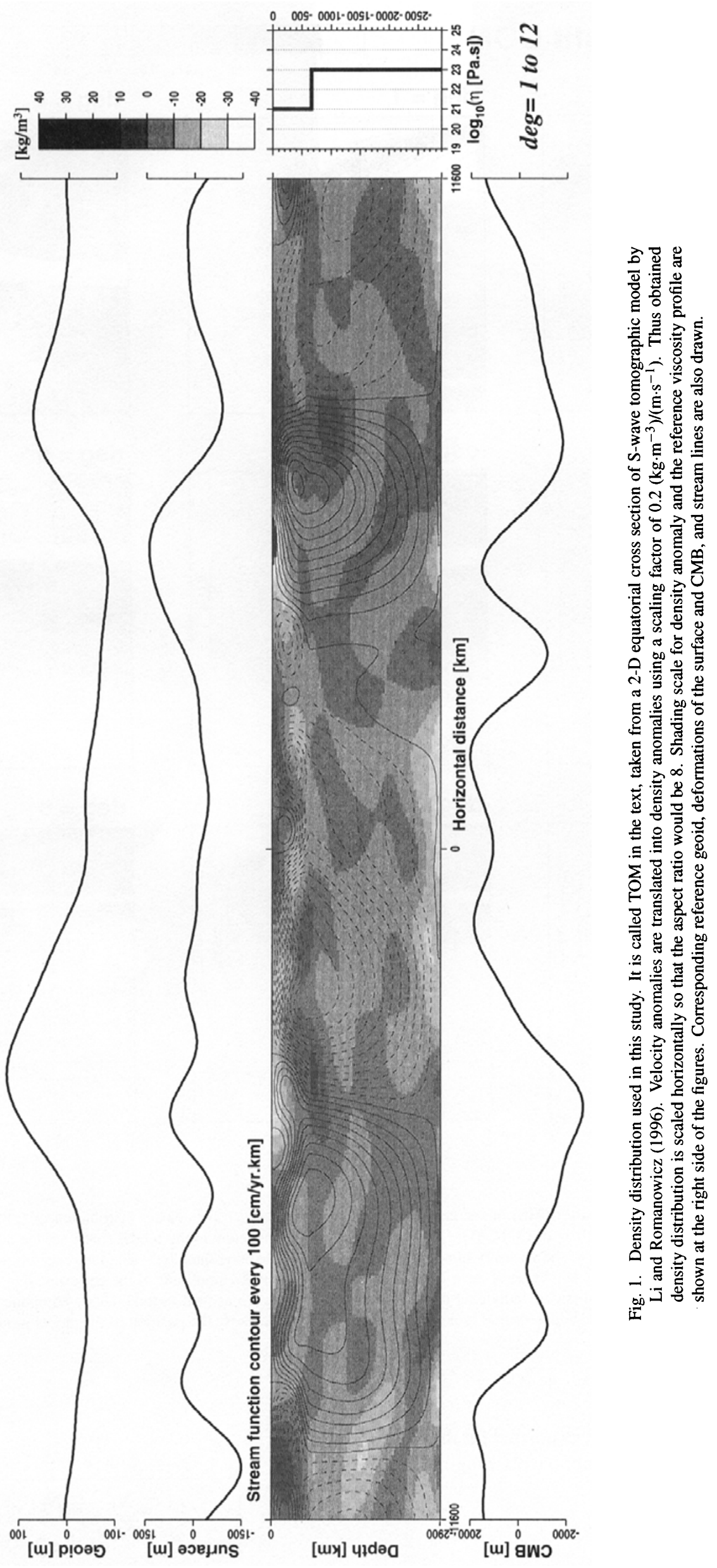




\section{(a) RMS geoid misfit CON}
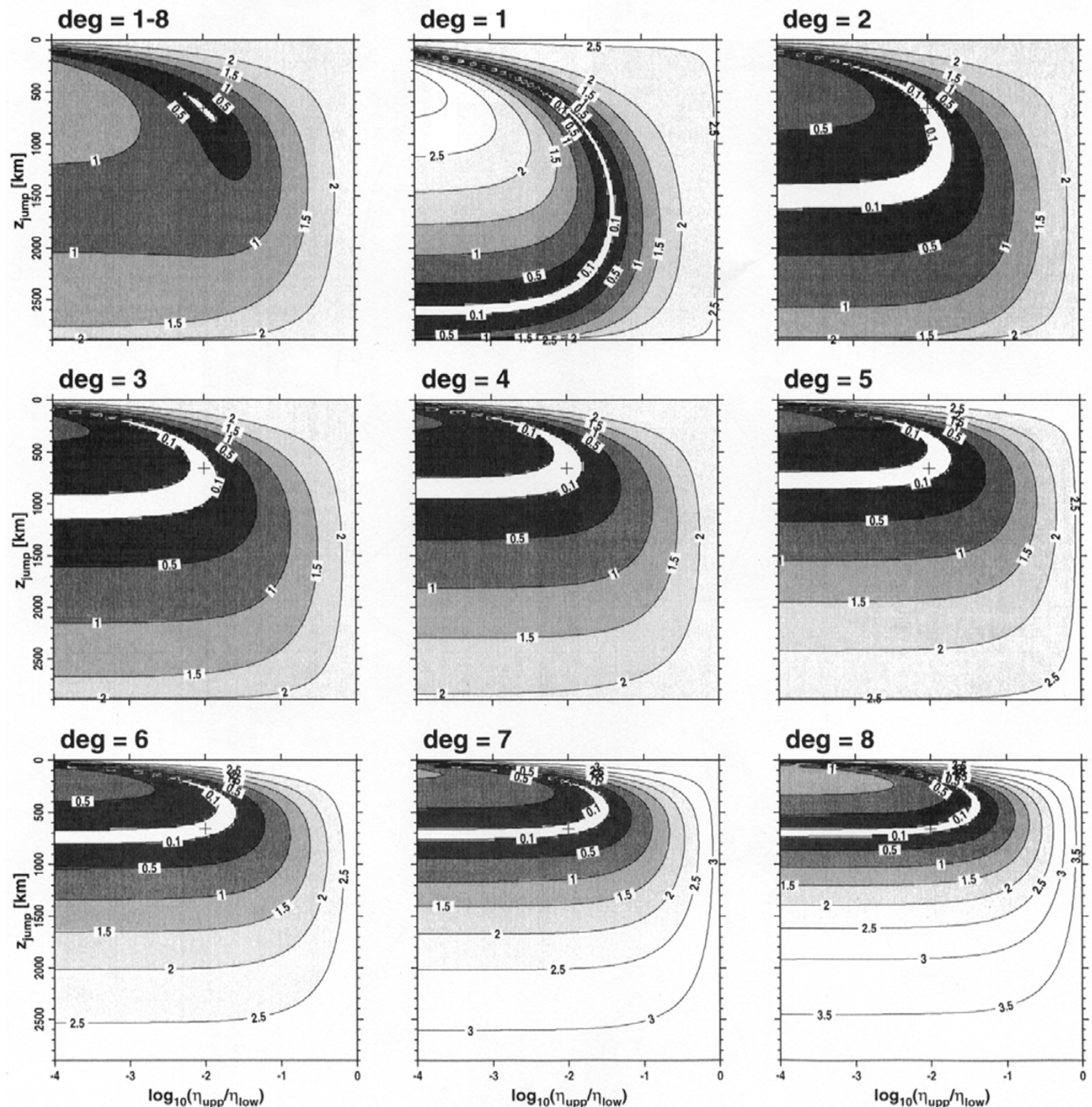

Fig. 2. Morphology of the root mean square (RMS) of the geoid misfit in 2-D model space for density distribution of (a) CON, (b) TOM, (c) T30, and (d) T70. See in the text for the notations of CON, TOM, T30, and T70. Two parameters in the model space are the depth of the viscosity jump $z_{\text {jump }}$ for the vertical axis and the amount of the viscosity jump $\log _{10}\left(\eta_{\text {upp }} / \eta_{\text {low }}\right)$ for the horizontal axis. The reference viscosity, $z_{\text {jump }}=660 \mathrm{~km}$ and $\log _{10}\left(\eta_{\text {upp }} / \eta_{\text {low }}\right)=-2$, is indicated by a "+". Value of the misfit is normalized by the RMS amplitude of the corresponding degree components of the reference geoid. For all the density distributions, individual degree components are shown as well as total degree components of 1-8, information of which is depicted at the top of each panel. Supplemental broken contours are added to clearify the position of the global minimum in the top left panel for $(\mathrm{c})$ and $(\mathrm{d})$

the reference geoid, called misfit, are evaluated for the entire model space. The misfit $D$ for components ranging from degree $\ell_{1}$ to $\ell_{2}$ is defined by

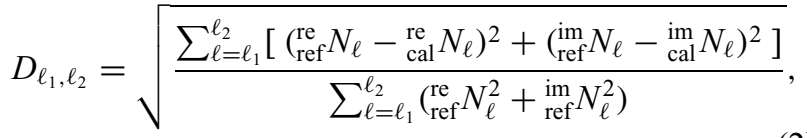




\section{(b) RMS geoid misfit TOM}
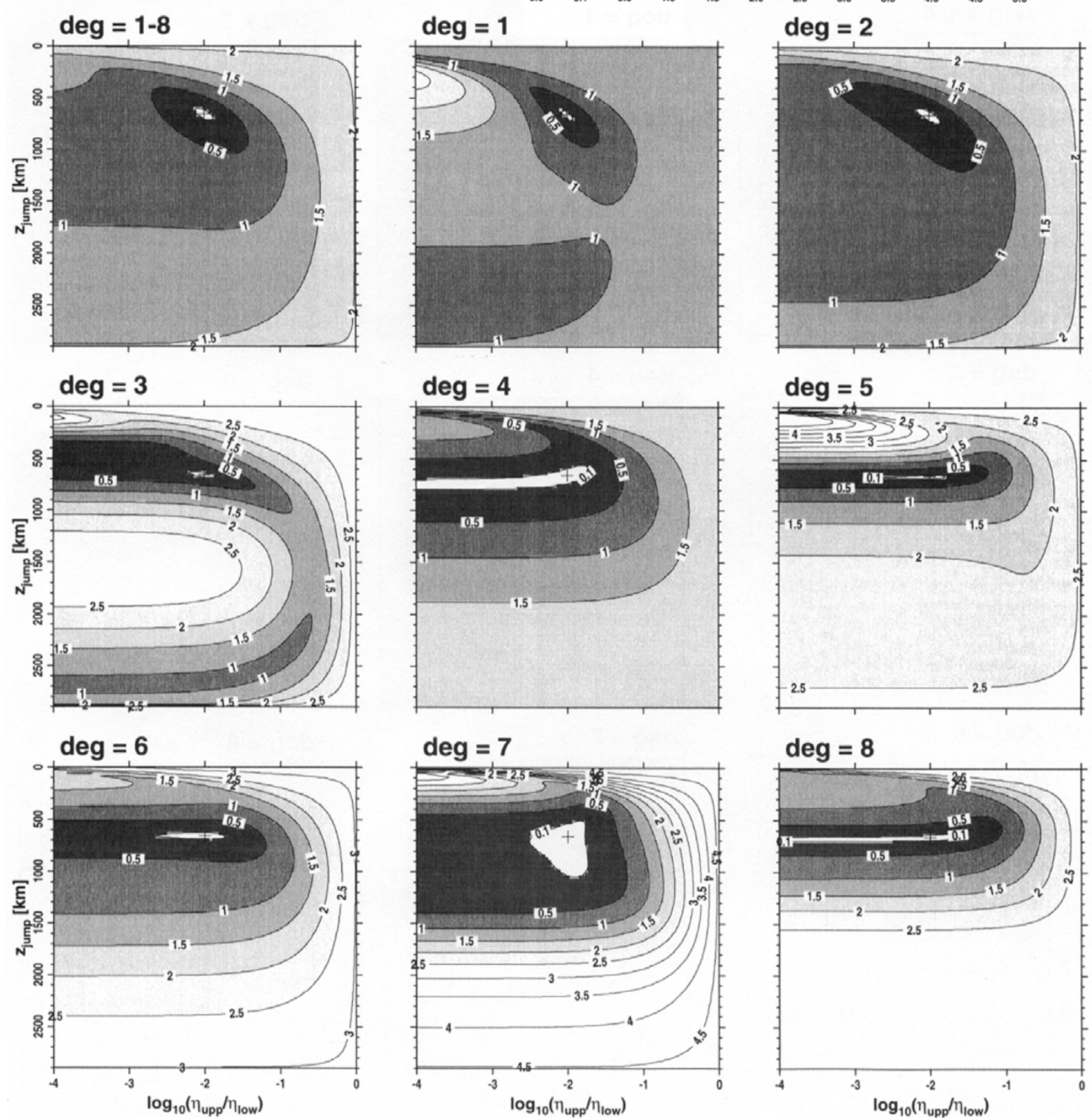

Fig. 2. (continued).

where "re" and "im" mean the real and imaginary parts of the geoid coefficients in the Fourier series.

We plotted a contour map of the misfit $D_{1,8}$ for the density distribution CON in the left top panel in Fig. 2(a). Vertical and horizontal axes represent the two parameters, $z_{\text {jump }}$ and $\log _{10}\left(\eta_{\text {upp }} / \eta_{\text {low }}\right)$ respectively, of the viscosity for calculating geoid. The position of the reference viscosity in the model space is indicated by a "+". The small misfit region where $D_{1,8}<0.1$ is shown in white surrounded by dark regions. Scale of the shadings is indicated by a shading bar at the top of the figure. We also plotted misfit for the individual degree component $D_{\ell}\left(=D_{\ell, \ell}\right.$ in Eq. (2)) in Fig. 2(a). Figures 2(b), 2(c) and 2(d) are the same as Fig. 2(a) but for the density distributions of TOM, T30, and T70, respectively.

Looking at the $D_{1,8}$ for CON in Fig. 2(a), the white area where $D_{1,8}<0.1$ is small and centered at the position of the reference viscosity. This indicates that the inversion will give an unique and correct viscosity solution. For the individual degree component of the misfit $D_{\ell}(\ell=1$ to 8$)$, on the other hand, we found that the white area tends to be stretched out and the shape of it systematically shifts upward with increase of degree while keeping the position of the reference 


\section{(c) RMS geoid misfit T30}
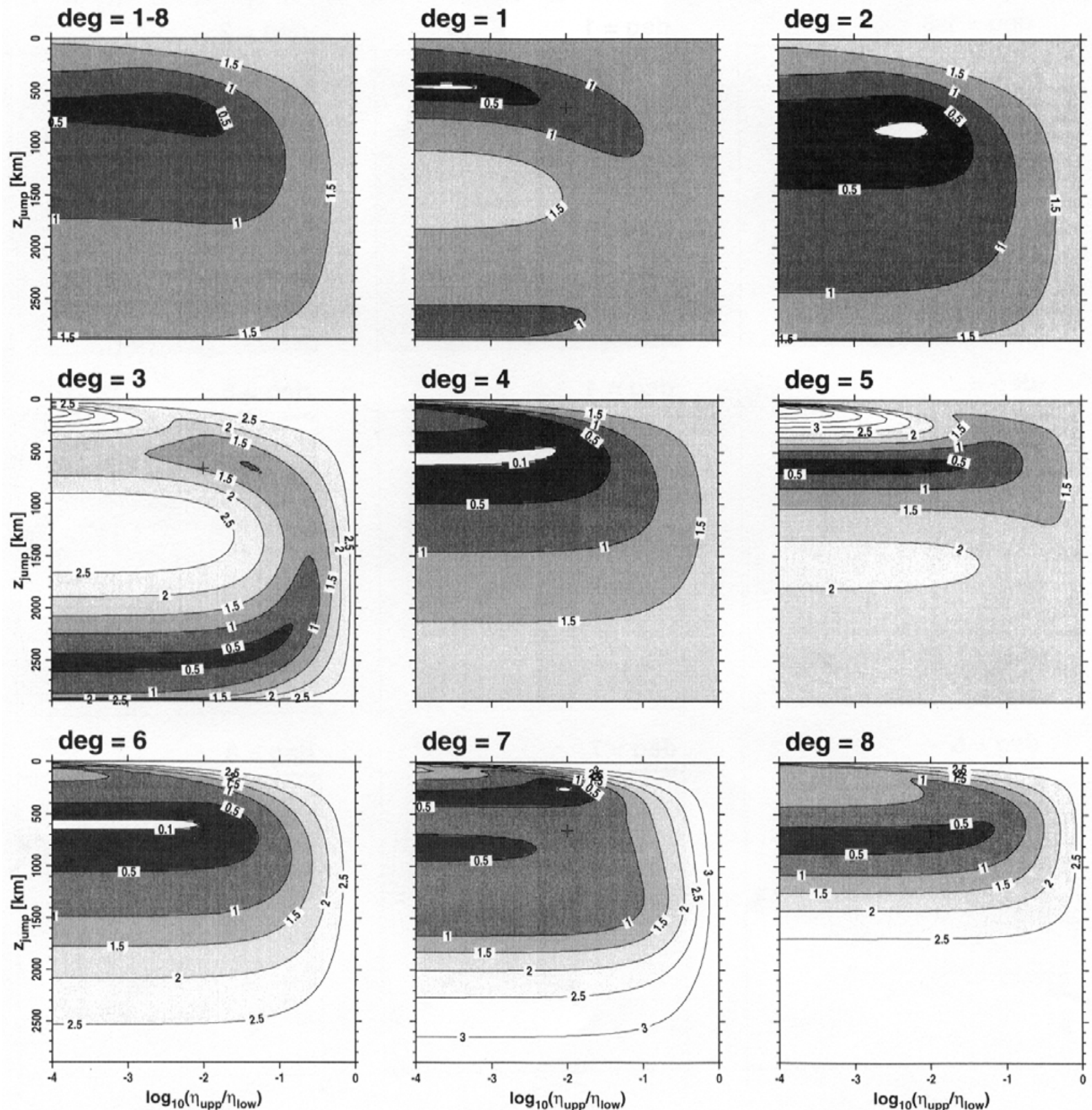

Fig. 2. (continued).

viscosity. This shows that a single wavelength component of geoid is not able to resolve the viscosity profile if density distribution has no vertical variation.

For the misfit $D_{1,8}$ for TOM in Fig. 2(b), the white area is also small as for the case CON. However, the behavior of the misfit for the individual degree components are quite different from that for CON. The systematic change of its morphology with degree is destroyed. The individual lower degree components, $D_{1}$ and $D_{2}$, already have strong constrains to the viscosity solution at least for this simple case of the two viscosity parameters. We observed a common feature with the case $\mathrm{CON}$ that higher degree components of the geoid are poor to resolve the $\log _{10}\left(\eta_{\text {upp }} / \eta_{\text {low }}\right)$. This is because the viscosity kernels for higher degrees are rather insensitive to such a large viscosity jump.

Finally in Figs. 2(c) and 2(d), we show the misfits for T30 and T70, containing 30\% and 70\% of noise defined by Eq. (1) in the density distribution. It should be noted that the misfit here is the difference between the reference geoid for TOM and calculated geoid for T30 or T70. Therefore the misfit does not necessarily to have zero area even at the position of the reference viscosity. However, some of the individual 


\section{(d) RMS geoid misfit T70}
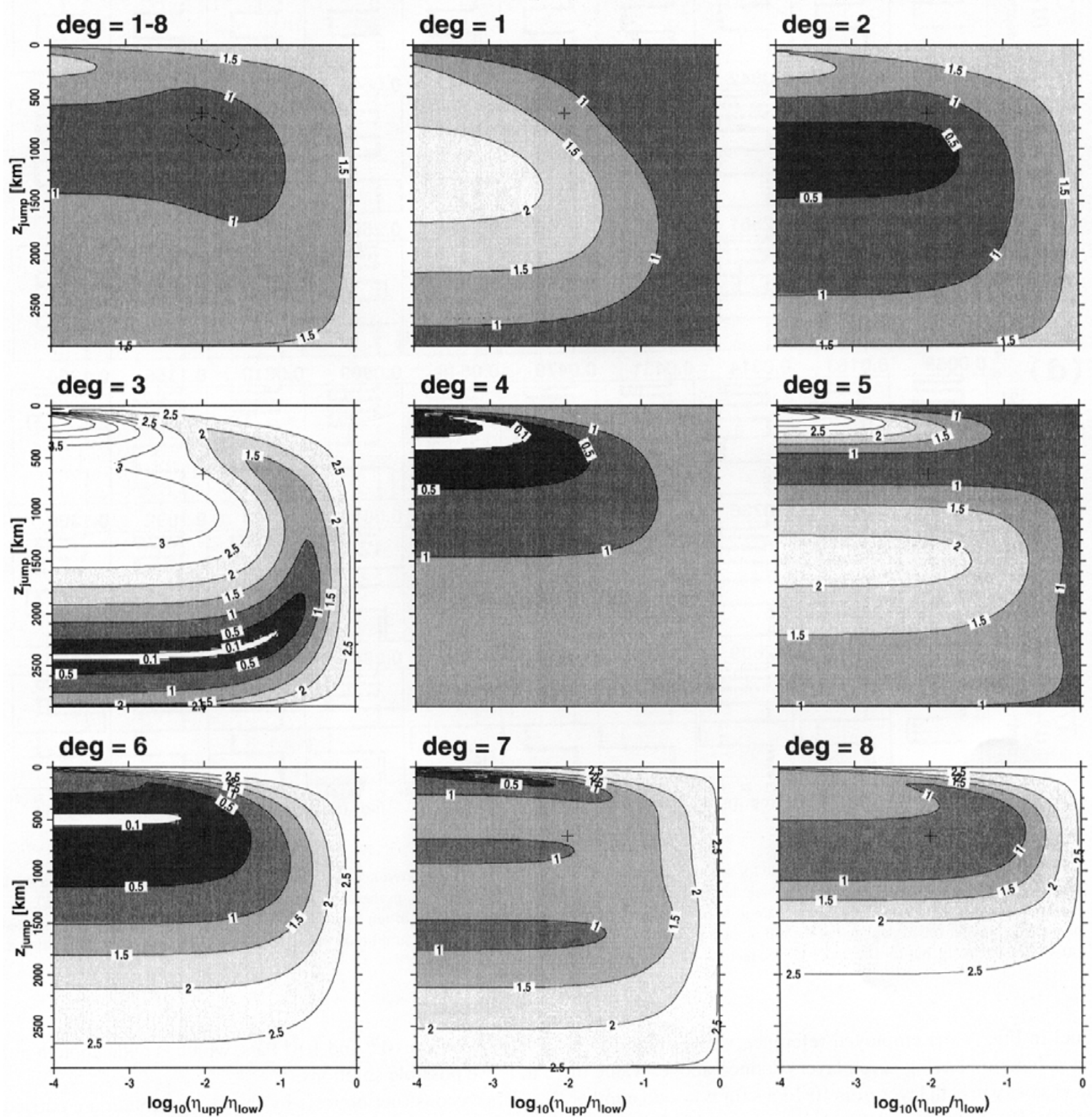

Fig. 2. (continued).

degree components of misfit has the white region even for large noise level T70 in Fig. 2(d). We should mention that all of these white regions do not coincide with the position of the reference viscosity. Especially for $D_{1}$ of T30 in Fig. 2(c) and $D_{3}$ of T70 in Fig. 2(d), white regions appear completely different position from the reference viscosity. Nevertheless such strange behavior of the misfit in the model space for the individual degree components, the global minimum of the misfit (indicated by supplemental contours) for the entire degree band $D_{1,8}$ is close to the position of the reference viscosity both for T30 and T70. This might be a kind of averaging effect.

\section{A Case for Six Parameters}

Now we examine the uniqueness of the inversion for a more realistic situation with six parameters describing the viscosity profile. Since the geoid is insensitive to the absolute value of the viscosity, we assume seven layers, where the bottom layer is fixed to be a constant viscosity value. Depth of the bottom of each layer is $164.93,379.35,658.08,1020.44,1491.51$, 2103.90 , and $2900.00 \mathrm{~km}$, where layer thickness is increasing with increase of its depth. As shown by a dotted line in any 


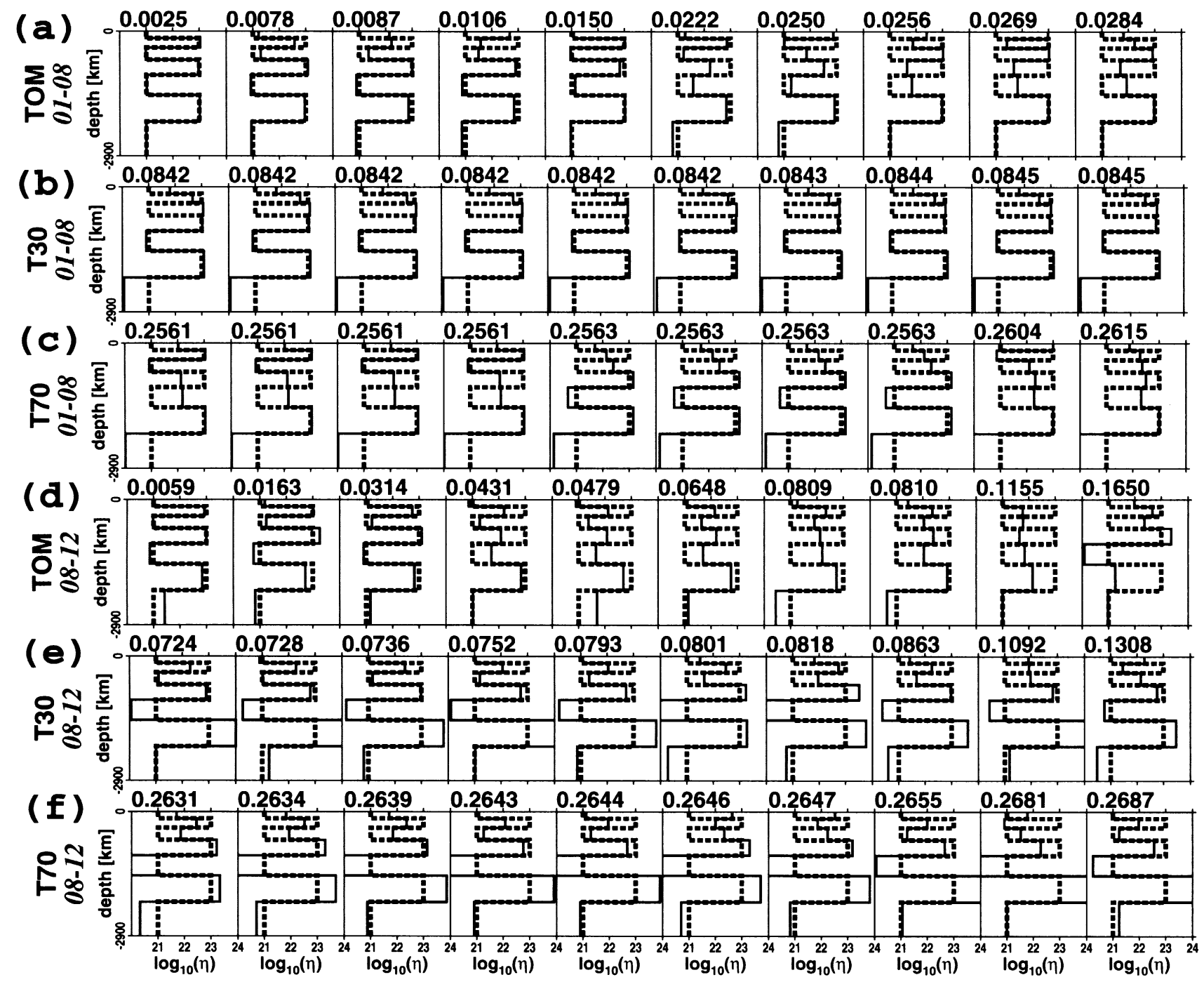

Fig. 3. Reconstruction tests of viscosity profile for seven layers case (six free parameters) from reference geoid for the reference viscosity drawn by a thick dotted line. The tests have been done for three density distributions TOM, T30, and T70. See in the text for the notations of TOM, T30, and T70. For (a)-(c), components of lower degrees 1-8 are used in the reconstruction. In each test, results of ten times of the inversion are shown, which are sorted along with smaller misfit depicted at the top of each panel. The misfit is normalized by RMS amplitude of the reference geoid. (d)-(f) are the same as (a)-(c), respectively, but for the upper degree band 8-12.

panel in Fig. 3, the employed reference viscosity profile is a step function having seven layers defined above. Value of the viscosity in $n$-th layer $\eta_{n}$ is $10^{21} \mathrm{~Pa} \cdot \mathrm{s}$ for $n=$ odd number and $10^{23} \mathrm{~Pa} \cdot \mathrm{s}$ for $n=$ even number, respectively. The density distributions used here are the same as CON, TOM, T30, and T70 in the two parameters case.

Since it is impossible to search the entire model space of geoid misfit as in the case of two parameters only, we try to reconstruct a reference viscosity from reference geoid using genetic algorithm (GA) inversion, which is suitable for a wide range search of the model space. As is written in Section 1, GA is much more efficient than Monte Carlo search while keeping access to the entire model space. Technical detail of the GA inversion used here is described in Kido et al. (1998). The GA inversion used here minimizes a misfit between the reference geoid for the reference viscosity and the calculated geoid for a model viscosity. For the model viscosity, each layer (except for the fixed bottom layer) is allowed to have a value between $10^{20}$ and $10^{24} \mathrm{~Pa} \cdot \mathrm{s}$, which is wide enough not to loose possible solutions.

The reconstruction tests by the GA inversion are carried out for lower degree band 1-8 and higher degree band 8-12, where 12 is the cut-off degree in the tomographic model. The results of the tests are shown in Fig. 3. Here, the results for the density distribution CON are omitted because we obtained no acceptable solution in the inversion for CON. We will mention about this in the next section. Then we have three density distributions TOM, T30, and T70 and each of them has results for lower (1-8) and higher (8-12) degree bands, so totally six rows of viscosity profiles are shown in Figs. 3(a)-3(f). The density distribution and the degree band are depicted at the left side of each row.

Each of the six tests consists of ten times of the GA inversion, results of which are drawn in an individual panel by a solid line superimposed on a dotted line of the reference viscosity. The panels are sorted in terms of smaller misfit 
depicted at the top of each panel, which is normalized by RMS amplitude of the reference geoid as was defined by Eq. (2). Here, it should be noted that viscosity profiles with considerably large misfit relative to the smallest one within a same test should not be counted as multiple solutions; this is due to the failure of the GA inversion and not due to the non-uniqueness in the model space.

For the density model TOM the best results for both the lower (Fig. 3(a)) and higher (Fig. 3(d)) degree bands have almost reconstructed the reference viscosity and their misfits are very small $(0.0025$ and 0.0059 for the lower and upper degree bands). However, the worse solutions are far from the reference viscosity though their misfits are still small. This means that there are trade-off among viscosity layers even for the noise-free density distribution.

Existence of the noise in density (T30 and T70) apparently devaluates the reconstruction even in the best results. The reason of this is that a local minimum of misfit in the model space does not coincide to the reference viscosity. This has been also observed in the two parameters case in Section 2 . Actually, misfits calculated for the reference viscosity are larger by a factor of two or three than the misfits of all the results for T30 and T70. The misfits calculated for the reference viscosity are 0.2377 for the lower band of T30, 0.4242 for the lower band of T70, 0.2188 for the upper band of T30, and 0.3940 for the upper band of T70. Results for T30 and T70 tend to converge to certain profiles, which are far from the reference viscosity, while solutions in TOM are oscillating near the reference viscosity.

\section{Discussion}

In this study, we performed uniqueness tests for the geoidviscosity inversion under various conditions. In general, the viscosity inversion is not a unique problem and has a large trade-off between viscosity parameters even using noise-free data. However, using numbers of wavelength components of geoid and fitting all of them simultaneously, the uniqueness of the inversion will be largely improved. How much improvement could be expected is owing to the noise content in the data being used. For the inversion of a viscosity profile, we can treat the observed geoid as noise free, whereas density data from seismic tomography has large uncertainties due to the interpretation in the seismic velocity anomalies, which can be of thermal or chemical origin. Therefore we used density data with rather large noise of $70 \%$. Here we interpret the results obtained in each section.

In Section 2, a model of only two viscosity layers was concerned to grasp general features of the effects of number of components and the noise in data on the uniqueness of viscosity solution. We employed unrealistic simple situations in order to see the behavior of trade-off, such as the use of vertically constant density distribution CON and analyses for individual degree component of geoid. In Fig. 2(a), all of the individual component of misfit have a sharp tradeoff between two parameters describing a viscosity profile. This is quite natural since there is only one component while freedom of this problem is two. It should be noted that the individual single degree analysis for TOM in Fig. 2(b) has two components, real and imaginary parts of the Fourier series. If we plotted real and imaginary parts of misfit separately, all of the misfits for individual degree for TOM has strong trade-off. Therefore individual degree components of misfit in Fig. 2(b) can have an unique solution, like $D_{1}$ and $D_{2}$, if combination of depth variation of the real and imaginary parts of the density distribution is appropriate. However, understanding the appropriate combination is difficult since geoid consists of the convolution of density variation and geoid kernel, which has nonlinear response to viscosity variation. We can only say that zero misfit or the white area of each single component in the model space has basically " $\supset$ "-figure as in Fig. 2(a) for any vertical density distribution. Rather complex morphology of the model space of individual degree for TOM in Fig. 2(b) comes from combination of two slightly different (real and imaginary) " $\supset$ " like morphologies. In this stand point, resolving power of higher degrees in the spherical 3-dimensional Earth is expected to have more unique morphology than for the 2-dimensional analysis. This is because spherical harmonics has many components of order as well as real and imaginary parts in a single degree.

We employed another density distribution containing artificial noise. Concerning the uncertainty in the interpretation of the seismic data (Jordan, 1978), or some unique use of higher degree components of tomographic data (Kido and Čadek, 1997), noise level was set to rather large value of $70 \%$ (T70) here. We also tested a case of moderate noise of $30 \%$ (T30). Even with the noise, some of the individual degree components of misfit have zero white region in the model space but it is not at a position of the reference viscosity. This means noise in the density data can mislead a solution of the inversion nevertheless misfit is very small or zero. Fortunately, using wide enough degree band 1-8, the peculiarity in each individual degree components smeared out and the global minimum in the misfit locates close to the reference viscosity (broken contours in Figs. 2(c) and 2(d)). In this case, misfits of the global minimum are large $(0.36$ for T30 and 0.72 for T70), which is not the matter with the inversion to obtain a plausible viscosity profile.

In Section 3, resolution tests were extended to a more realistic case with six viscosity parameters. As was mentioned above, logical comprehension about the morphology of the model space is complicated even for the two viscosity parameters case. Therefore we have to empirically recognize its behavior by the model reconstruction and the value of the misfit. We could reconstruct the reference viscosity in a practical level at least for the best result out of ten times of inversion for noise-free density distribution TOM. For the density distribution with noise (T30 and T70), solutions are stable, however, reconstruction of the reference viscosity was failed. There are two possible reasons for the failure of the reconstruction. One is the non-uniqueness of the model space itself and the other is a technical problem in the GA inversion. Looking at the Figs. 3(a) and 3(d), the noise free density distribution TOM, values of the misfit vary among ten times of the inversion, though they are still small. In this case, the latter is the cause of the poor reconstruction due to rather flat morphology near the global minimum of misfit in the model space. On the contrary, for T30 and T70, solutions are stable and hence values of the misfit are nearly constant for most of the ten times of the GA inversion. This would be the former case, since the inversions have reached 
to the rather sharp global minimum, which is not close to the reference viscosity. There is a special case that results shown in Fig. 3(c) have three independent local minima having nearly the same misfit. This lead the inversion to result in the multiple solutions.

We have applied various types of reference viscosity for the six viscosity parameter tests, having an opposite sign or smaller oscillation. In addition, density distributions of no vertical variation (called CON in Section 2) or of little vertical variation. Results of them are not shown in this paper. Particular feature of their results are different to one another, however, we found that a part of the descriptions in the previous paragraph can be common behavior. They are; (1) there is a rather flat hill at the position of the reference viscosity for a density distribution without noise, (2) there is a sharp global minimum which lies apart from the reference viscosity for a density distribution with noise, which split into a few peaks in some cases. Only the exception is the case for CON, which has too many solutions of zero misfit, hence the inversion does not make sense. For the density distribution with the noise, misfits of obtained solutions are rather large compared to that for the noise-free density distribution, but are still smaller than those calculated by the reference viscosity. This is why they result in solutions different from the reference viscosity.

In this study, we tested resolving power of the geoidviscosity inversion mainly on the effect of noise in the density data. However, we have many other problems when we apply the inversion to the real Earth, such as validity of viscosity representation by several iso-viscous layers, neglecting the lateral viscosity variation in the mantle, and so on. To examine the exact resolving power of the geoid-viscosity inversion, these problems must be considered simultaneously in a realistic spherical geometry. One should conduct synthetic tests like presented in this study with his particular conditions in order to confirm reliability of their inversion before applying the geoid-viscosity inversion to the Earth. We should also mention that obtaining a small misfit has no warranty for the validity of the solution in the geoid-viscosity inversion. If the problem is not unique or has distorted global minimum due to noise in data, one can obtain small or even zero misfit with a wrong viscosity solution. Important thing is whether the solution is unique and lies near the real viscosity. Though we have an interest in theoretical analysis of the model space, it is difficult to elucidate the relation between vertical distribution of the density and model space morphology for individual degree components. However the peculiar behavior of a single component would be smeared out by using spherical harmonics, which has much more components in a single degree. This enable us to understand the statistical systematics in the resolving power of the geoid-viscosity inversion.

Acknowledgments. We thank Dr. Bertram Schott for reading the entire manuscript and providing valuable suggestions. Comments by Dr. Alessandro M. Forte and two anonymous reviewers were helpful to improve this paper. Tomographic data used in this study is provided by Dr. Barbara Romanowicz. This work has been supported by JSPS 9706773.

\section{References}

Čadek, O., Y. Ricard, Z. Martinec, and C. Matyska, Comparison between Newtonian and non-Newtonian flow driven by internal loads, Geophys. J. Int., 112, 103-114, 1993.

Čadek, O., H. Čížková, and D. A. Yuen, Can longwavelength dynamical signatures be compatible with layered mantle convection?, Geophys. Res. Lett., 24, 2091-2094, 1997.

Corrieu, V., C. Thoraval, and Y. Ricard, Mantle dynamics and geoid Green functions, Geophys. J. Int., 120, 516-523, 1995.

Doin, M.-P., L. Fleitout, and D. P. McKenzie, Geoid anomalies and the structure of continental and oceanic lithospheres, J. Geophys. Res., 101, 16119-16135, 1996

Forte, A. M. and W. R. Peltier, Viscous flow models of global geophysical observables 1. Forward problems, J. Geophys. Res., 96, 20131-20159, 1991.

Forte, A. M. and W. R. Peltier, The kinematics and dynamics of poloidaltoroidal coupling in the mantle flow: The importance of surface plates and lateral viscosity variations, Adv. Geophys., 36, 1-119, 1994.

Forte, A. M. and R. L. Woodward, Global 3D mantle structure and vertical mass and heat transfer across the mantle from joint inversions of seismic and geodynamic data, J. Geophys. Res., 102, 17981-17994, 1997.

Forte, A. M., R. L. Woodward, and A. M. Dziewonski, Joint inversions of seismic and geodynamic data for models of three-dimensional mantle heterogeneity, J. Geophys. Res., 99, 21857-21877, 1994.

Forte, A. M., A. M. Dziewonski, and R. J. O'Connell, Thermal and chemical heterogeneity in the mantle: A seismic and geodynamic study of continental roots, Phys. Earth Planet. Inter., 92, 45-55, 1995.

Goldberg, D. E., Genetic Algorithms in Search, Optimization, and Machine Learning, pp. 412, Addison-Wesley Publishing Company, Inc., 1989.

Hager, B. H. and W. R. Clayton, Constraints on the structure of mantle convection using seismic observations, flow models and the geoid, in Mantle Convection, edited by W. R. Peltier, pp. 657-763, Pergamon Press, 1989.

Hager, B. H. and M. A. Richards, Long-wavelength variations in Earth's geoid: physical models and dynamical implications, Phil. Trans. R. Soc. Lond., A328, 309-327, 1989.

Jordan, T. H., Composition and development of the continental tectosphere, Nature, 274, 544-548, 1978.

Karato, S., Effects of water on seismic wave velocities in the upper mantle, Proc. Japan. Acad., 71, Ser. B, 61-66, 1995.

Kido, M. and O. Čadek, Inferences of viscosity from the oceanic geoid: Indication of a low viscosity zone below the $660-\mathrm{km}$ discontinuity, Earth Planet. Sci. Lett., 151, 125-137, 1997.

Kido, M., D. A. Yuen, O. Čadek, and T. Nakakuki, Mantle viscosity derived by genetic algorithm using oceanic geoid and seismic tomography for whole-mantle versus blocked-flow situations, Phys. Earth Planet. Inter, 151, 503-525, 1998.

King, S. D., Radial models of mantle viscosity: results from a genetic algorithm, Geophys. J. Int., 122, 725-734, 1995.

King, S. D. and G. Masters, An inversion for radial viscosity structure using seismic tomography, Geophys. Res. Lett., 19, 1551-1554, 1992.

Li, X.-D. and B. Romanowicz, Global mantle shear velocity model developed using nonlinear asymptotic coupling theory, J. Geophys. Res., 101, 22245-22272, 1996

Mitrovica, J. X. and A. M. Forte, Radial profile of mantle viscosity: Results from the joint inversion of convection and postglacial rebound observables, J. Geophys. Res., 102, 2751-2769, 1997.

Panasyuk, S. V., B. H. Hager, and A. M. Forte, Understanding the effects of mantle compressibility on geoid kernels, Geophys. J. Int., 124, 121-133, 1996.

Ribe, N. M., The dynamics of thin shells with variable viscosity and the origin of toroidal flow in the mantle, Geophys. J. Int., 110, 537-552, 1992.

Ricard, Y., C. Froidevaux, and L. Fleitout, Global plate motion and the geoid: a physical model, Geophys. J., 93, 477-484, 1988.

Ricard, Y., C. Vigny, and C. Froidevaux, Mantle heterogeneities, geoid, and plate motion: A Monte Carlo inversion, J. Geophys. Res., 94, 13739_ 13754, 1989.

Ricard, Y., C. Doglioni, and R. Sabadini, Differential rotation between lithosphere and mantle: A consequence of lateral mantle viscosity variation, J. Geophys. Res., 96, 8407-8415, 1991.

Ricard, Y., M. Richards, C. Lithgow-Bertelloni, and Y. Le Stunff, A geodynamic model of mantle density heterogeneity, J. Geophys. Res., 98 21895-21909, 1993

Ricard, Y., H.-C. Nataf, and J.-P. Montagner, The 3-SMAC model: Con- 
frontation with seismic data, J. Geophys. Res., 1995 (submitted).

Richards, M. A. and B. H. Hager, Geoid anomalies in a dynamic earth, $J$ Geophys. Res., 89, 5987-6002, 1984.

Richards, M. A. and B. H. Hager, Effects of lateral viscosity variation on long-wavelength geoid anomalies and topography, J. Geophys. Res., 94, 10299-10313, 1989.

Sen, M. K. and P. L. Stoffa, Rapid sampling of model space using genetic algorithms: examples from seismic waveform inversion, Geophys. J. Int., 108, 281-292, 1992.

Tarantola, A. and B. Valette, Generalized nonlinear inverse problems solved using the least squares criterion, Rev. Geophys. Space Phys., 20, 219-232, 1982.

Thoraval, C., Ph. Machetel, and A. Cazenave, Influence of mantle compressibility and ocean warping on dynamical models of the geoid, Geophys. $J$.
Int., 117, 566-573, 1994.

Thoraval, C., Ph. Machetel, and A. Cazenave, Locally layered convection inferred from dynamic models of the Earth's mantle, Nature, 375, 777789, 1995.

Wen, L. and D. L. Anderson, Layered mantle convection: A model for geoid and topography, Earth Planet. Sci. Lett., 146, 367-378, 1997.

Zhang, S. and U. R. Christensen, The effect of lateral viscosity variations on geoid, topography and plate motions induced by density anomalies in the mantle, Geophys. J. Int., 114, 531-547, 1993.

M. Kido (e-mail: kido@ori.u-tokyo.ac.jp) and S. Honda (e-mail: satoru@geol.sci.hiroshima-u.ac.jp) 
Fig. 1. Density distribution used in this study. It is called TOM in the text, taken from a 2-D equatorial cross section of S-wave tomographic model by $\mathrm{Li}$ and Romanowicz (1996). Velocity anomalies are translated into density anomalies using a scaling factor of $0.2\left(\mathrm{~kg} \cdot \mathrm{m}^{-3}\right) /\left(\mathrm{m} \cdot \mathrm{s}^{-1}\right)$. Thus obtained density distribution is scaled horizontally so that the aspect ratio would be 8 . Shading scale for density anomaly and the reference viscosity profile are shown at the right side of the figures. Corresponding reference geoid, deformations of the surface and CMB, and stream lines are also drawn. 